\title{
Changes in the circumscription of Deprea (Physalideae, Solanaceae): thirty two new combinations
}

\author{
Rocío Deanna ${ }^{1,3}$, Segundo Leiva González², Gloria Estela Barboza ${ }^{1,3}$ \\ I Instituto Multidisciplinario de Biologia Vegetal (IMBIV), CONICET and Universidad Nacional de Córdoba, \\ CC 495, CP 5000, Córdoba, Argentina 2 Museo de Historia Natural, Universidad Privada Antenor Orrego de \\ Trujillo, CC 1075, Trujillo, Perú 3 Facultad de Ciencias Químicas, Universidad Nacional de Córdoba, Haya \\ de la Torre y Medina Allende, Córdoba, Argentina
}

Corresponding author: Gloria Estela Barboza (gbarboza@imbiv.unc.edu.ar)

Academic editor: Sandra Knapp | Received 4December 2014 | Accepted 10 Febtuary 2015 | Published 27 February 2015

Citation: Deanna R, Leiva González S, Barboza GE (2015) Changes in the circumscription of Deprea (Physalideae, Solanaceae): thirty two new combinations. PhytoKeys 46: 73-87. doi: 10.3897/phytokeys.46.9069

\begin{abstract}
According to the latest phylogenetic and cytogenetic results, Larnax and Deprea should be merged in order to form a natural group. Consequently, we propose 32 combinations of Larnax species names under Deprea: D. abra-patriciae (S.Leiva \& Barboza) S.Leiva \& Deanna, comb. nov., D. altomayoensis (S.Leiva \& Quip.) Barboza \& Deanna, comb. nov., D. andersonii (N.W.Sawyer) Deanna \& S.Leiva, comb. nov., D. bongaraensis (S.Leiva) Deanna \& Barboza, comb. nov., D. chotanae (S.Leiva, Pereyra \& Barboza) S.Leiva, comb. nov., D. darcyana (N.W.Sawyer) Barboza \& S.Leiva, comb. nov., D. dilloniana (S.Leiva, Quip. \& N.W.Sawyer) Barboza, comb. nov., D. grandiflora (N.W.Sawyer \& S.Leiva) Deanna \& Barboza, comb. nov., D. harlingiana (Hunz. \& Barboza) S.Leiva \& Deanna, comb nov., D. hawkesii (Hunz.) Deanna, comb. nov., D. kann-rasmussenii (S.Leiva \& Quip.) S.Leiva \& Barboza, comb. nov., D. longipedunculata (S.Leiva, E.Rodr. $\&$ J.Campos) Barboza, comb. nov., D. lutea (S.Leiva) Deanna, comb. nov., D. macasiana (Deanna, S.Leiva \& Barboza) Barboza, comb. nov., D. maculatifolia (E.Rodr. \& S.Leiva) S. Leiva, comb. nov., D. nieva (S.Leiva \& N.W.Sawyer) Barboza \& Deanna, comb. nov., D. parviflora (N.W.Sawyer \& S.Leiva) S.Leiva, comb. nov., D. pedrazae (S.Leiva \& Barboza) Deanna \& S.Leiva, comb. nov., D. peruviana (Zahlbr.) S.Leiva $\&$ Barboza, comb. nov., D. pilosa (S.Leiva, E.Rodr. \& J.Campos) Deanna, comb. nov., D. pomacochaensis (S.Leiva) Barboza, comb. nov., D. psilophyta (N.W.Sawyer) S.Leiva \& Deanna, comb. nov., D. pumila (S.Leiva, Barboza \& Deanna) S.Leiva, comb. nov., D. purpurea (S.Leiva) Barboza \& S.Leiva, comb. nov., D. purpureocarpa (S.Leiva, Deanna \& Barboza) Deanna, comb. nov., D. sachapapa (Hunz.) S.Leiva \& Deanna, comb. nov., D. sagasteguii (S.Leiva, Quip. \& N. W.Sawyer) Barboza, comb. nov., D. sawyeriana (S.Leiva, E.Rodr. \& J.Campos) S.Leiva, comb. nov., D. schjellerupiae (S.Leiva \& Quip.) Barboza \& Deanna, comb. nov., D. steyermarkii (Hunz.) S.Leiva \& Barboza, comb. nov., D. toledoana (Barboza \& S.Leiva) Barboza, comb. nov., and D. vasquezii (S.Leiva, E.Rodr. \& J.Campos) Deanna, comb. nov.
\end{abstract}

Copyright Rocío Deanna et al. This is an open access article distributed under the terms of the Creative Commons Attribution License (CC BY 4.0), which permits unrestricted use, distribution, and reproduction in any medium, provided the original author and source are credited. 


\section{Keywords}

Deprea, new combinations, Solanaceae, Larnax

\section{Introduction}

Larnax Miers and Deprea Raf. are two closely related genera of Physalideae tribe (Solanaceae) that include 35 and 10 species, respectively. Both genera are restricted to the neotropics mainly inhabiting pre-montane and montane cloud forest from Bolivia to Costa Rica. Both Larnax and Deprea include species ranging from herbs to small trees with funnel-shaped to stellate corollas, but always with accrescent fruiting calyces tightly or loosely enclosing the berries (Sawyer 2005, Deanna et al. 2014a). The main centre of diversity for both genera is Peru and Ecuador (Leiva González et al. 2008, Deanna et al. 2014a, Jørgensen and León-Yánez 1999). Only one taxon extends north into Central America [L. sylvarum subsp. sylvarum (Standl. \& C.V.Morton) N.W.Sawyer, Sawyer 2001] and one species is found at the south of their range in Bolivia [L. subtriflora (Ruiz \& Pav.) Miers, Leiva González et al. 2013].

Deprea and Larnax are interesting for their chemical and medicinal value (Cardona et al. 2005, Misico et al. 2011, Casero et al. 2015), but also for their controversial circumscription and position within the Physalideae tribe (Olmstead et al. 2008, Särkinen et al. 2013, Carrizo García et al. submitted). Various authors have transferred species between the two genera for many years (Miers 1849, D’Arcy 1973, 1993, Sawyer 2001, Leiva González et al. 2005) and redefined their limits (Hunziker 2001, Sawyer 2005). Sawyer (2005) differentiated Deprea and Larnax based on six characters (filament base expansion, filament length, filament adnation, anther length, corolla shape, and pollen surface texture) according to morphological cladistic work, although some characters overlap in the two genera. Moreover, Sawyer (1999) obtained ambiguous results when attempting to set apart these genera using DNA sequence data in cladistic analysis, and the most recent phylogenetic analysis of the family (Särkinen et al. 2013) suggests they are not monophyletic.

Here we deal with the new circumscription of the two genera under Deprea and the consequent new combinations of Larnax based on recent phylogenetic evidence that show Deprea and Larnax to be monophyletic when their species are merged (100\% bootstrap support), where species, as L. sachapapa Hunz., are resolved nested within a group of Deprea species, or vice versa (Carrizo et al. submitted). The phylogenetic evidence is further supported by results from recent extensive field collecting and taxonomic work which have shown that characters utilized to distinguish the two genera are not useful, and increasing number of species are found to have intermediate features between the two genera (see Table 1, Deanna et al. 2014b; Carrizo et al. submitted). Consequently, this paper synonymises Larnax under Deprea, expands the morphological circumscription of Deprea, and transfers the remaining Larnax species to Deprea, presenting all 32 new combinations, and a list of all currently accepted 45 names in Deprea. Type specimens are also cited with their corresponding barcode number if it is available or their sheet number where it is not (HUT, QCA, QCNE, QUSF, and W). 


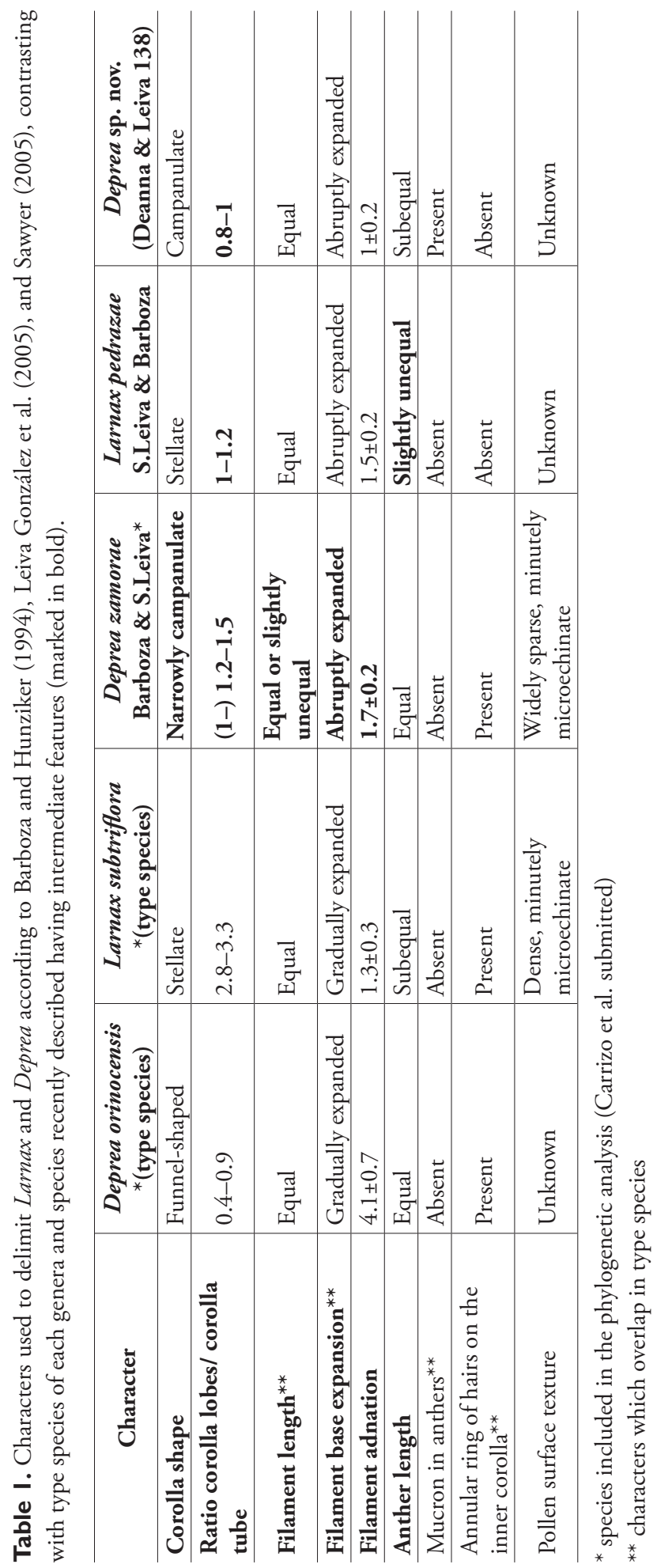




\section{Systematics}

\section{Deprea Raf., Sylva Tellur. 57. 1838.}

Larnax Miers, Ann. Mag. Nat. Hist., ser. 2 (4): 37. 1849, syn. nov.

Type. Deprea orinocensis (Kunth) Raf. (Lectotype species, designated in D'Arcy 1973 [1974]. Flora of Panama, Part IX. Family 170. Solanaceae. Ann. Missouri Bot. Gard. 60 (3): 624).

Erect shrubs to small trees with spreading branches, exceptionally herbs with axillary inflorescences generally with three to 15 flowers per node, calyx lobes minute or short, exceptionally long and narrowly triangular, corolla funnel-shaped to stellate, stamen petalum broadening gradually to abruptly in width basipetally, with or without auricles, anthers dorsifixed, generally exserted and mucronate, ovary glabrous, fruiting calyx always accrescent, enveloping the fleshy berry tightly or loosely.

Deprea abra-patriciae (S.Leiva \& Barboza) S.Leiva \& Deanna, comb. nov. urn:Isid:ipni.org:names:77145416-1

Basionym: Larnax abra-patriciae S.Leiva \& Barboza, Arnaldoa 16(1): 30. 2009.

Type. PERU. Amazonas: Bongará, Área de Conservación Privada Abra Patricia-Alto Nieva, km 364-365, carretera Fernando Belaunde Terry, 2250 m, 541'28.2"S, 7748'41.1"W, 16 May 2009 (fl, fr), S.Leiva 4561 (lectotype, designated in Deanna et al. 2014a, pg. 19: Leiva González \& Barboza, Arnaldoa 16(1): 32. Fig. 1. 2009).

\section{Deprea altomayoensis (S.Leiva \& Quip.) Barboza \& Deanna, comb. nov.} urn:Isid:ipni.org:names:77145417-1

Basionym: Larnax altomayoensis S.Leiva \& Quip., Arnaldoa 15 (2): 199. 2009 [2008 publ. Jan 2009].

Type. PERU. San Martin: Rioja, Bosque de Protección de Alto Mayo, $1010 \mathrm{~m}$, 540'11.5"S, 77³7'48.5"W, 24 July 2008 (fl, fr), S.Leiva, M.Zapata \& V.Quipuscoa 4480 (lectotype, designated in Deanna et al. 2014a, pg. 21: Leiva González, Pereyra Villanueva \& Barboza, Arnaldoa 15(2): 200. Fig. 1. 2008). 
Deprea andersonii (N.W.Sawyer) Deanna \& S.Leiva, comb. nov. urn:lsid:ipni.org:names:77145432-1

Basionym: Larnax andersonii N.W.Sawyer, Novon 8 (1): 72.1998.

Type. ECUADOR. Napo: km 25 of Hollín-Loreto road, finca entrance next to bridge over a quebrada in secondary pluvial forest, $950 \mathrm{~m}, 00^{\circ} 40^{\prime} \mathrm{S}, 77^{\circ} 40^{\prime} \mathrm{W}, 1 \mathrm{July} 1995$ (f, fr), N.W.Sawyer \& M.Tirado 714 (holotype: MO n.v.; isotypes: CONN! [00054622], QCNE! [105520], US n.v.).

Deprea bongaraensis (S.Leiva) Deanna \& Barboza, comb. nov. urn:lsid:ipni.org:names:77145418-1

Basionym: Larnax bongaraensis S.Leiva, Arnaldoa 13 (2): 292. 2006.

Type. PERU. Amazonas: Bongará, km 328-329, carretera Pomacochas-Nuevo Cajamarca, arriba de laguna Pomacochas, 2400 m, 5 Feb 2006 (fl, fr), S.Leiva 3543 (lectotype, designated in Deanna et al. 2014a, pg. 21: Leiva González \& Rodríguez Rodríguez, Arnaldoa 13(2): 293. Fig. 1. 2006).

Deprea chotanae (S.Leiva, Pereyra \& Barboza) S.Leiva, comb. nov. urn:lsid:ipni.org:names:77145419-1

Basionym: Larnax chotanae S.Leiva, Pereyra \& Barboza, Arnaldoa 15 (2): 203. 2008.

Type. PERU. Cajamarca: Chota, Bosque El Pargo, 3050 m, 30 Sep 2004 (fl, fr), S.Leiva \& J. Guevara 2883 (lectotype, designated in Deanna et al. 2014a, pg. 21: Leiva González, Pereyra Villanueva \& Barboza, Arnaldoa 15(2): 204. Fig. 3. 2008).

Deprea darcyana (N.W. Sawyer) Barboza \& S.Leiva, comb. nov. urn:lsid:ipni.org:names:77145433-1

Basionym: Larnax darcyana N.W.Sawyer, Novon 11 (4): 463. 2001.

Type. COLOMBIA. Huila: forest around Mehrenberg, road from Popayan, $2350 \mathrm{~m}$, 6 July 1984 (fl, fr), W.G.D 'Arcy, A.Gentry, M.Monsalve \& P.Silverstone 15626 (holotype: $\mathrm{MO}$ n.v.; isotype: CONN! [00054625]). 
Deprea dilloniana (S.Leiva, Quip. \& N.W.Sawyer) Barboza, comb. nov. urn:Isid:ipni.org:names:77145420-1

Basionym: Larnax dilloniana S.Leiva, Quip. \& N.W.Sawyer, Arnaldoa 5 (1): 85. 1998.

Type. PERU. San Martín: Rioja, arriba del poblado Miraflores (ca. Nueva Cajamarca), 1260-1420 m, 3 Nov 1996 (fl, fr), S.Leiva, M.Dillon, I.Sánchez, V.Quipuscoa \& P.Lezama 1919 (lectotype, designated in Deanna et al. 2014a, pg. 21: CONN! [00066074]; isolectotypes: CONN! [00066075, 00168924!], CORD! [00004043], F! [2183234], HUT! [031930], MO! [05097641]).

Deprea grandiflora (N.W.Sawyer \& S.Leiva) Deanna \& Barboza, comb. nov. urn:lsid:ipni.org:names:77145434-1

Basionym: Larnax grandiflora N.W.Sawyer \& S.Leiva, Novon 11 (4): 463. 2001.

Type. PERU. Cajamarca: San Ignacio, in forest along road from San Martín to El Chaupe, $1700 \mathrm{~m}, 05^{\circ} 11^{\prime} \mathrm{S}, 79^{\circ} 03^{\prime} \mathrm{W}, 26$ June 1997 (fl, fr), N. W.Sawyer 827 (holotype: NY!; isotypes: CONN! [00054628], MO n.v.).

Deprea harlingiana (Hunz. \& Barboza) S.Leiva \& Deanna, comb. nov. urn:lsid:ipni.org:names:77145435-1

Basionym: Larnax harlingiana Hunz. \& Barboza, Kurtziana 24: 157. 1995.

Type. ECUADOR. Zamora-Chinchipe: Nudo de Sabanilla, E slope c. $5 \mathrm{~km}$ from pass on road Yanganá-Valladolid, 2700 m, 4 Apr 1985 (fl, fr), G.Harling \& L.Andersson 23646 (holotype: GB! [0008131]; isotypes: CORD! [00006738], QCA! [156713]).

Deprea hawkesii (Hunz.) Deanna, comb. nov. urn:lsid:ipni.org:names:77145436-1

Basionym: Larnax hawkesii Hunz., Kurtziana 10: 9. 1977.

Type. COLOMBIA. Huila: Carretera a La Plata, región de Moscopán, Santa Leticia, 2230 m, 21 July 1948 (fl, fr), H.Garcia-Barriga \& J.C.Hawkes 12919 (holotype: US! [00385927]; isotypes: COL! [000004223, 000004224]). 
Deprea kann-rasmussenii (S.Leiva \& Quip.) S.Leiva \& Barboza, comb. nov. urn:lsid:ipni.org:names:77145437-1

Basionym: Larnax kann-rasmussenii S.Leiva \& Quip., Arnaldoa 9 (1): 29. 2002 [2003].

Type. PERU. San Martín: Huallaga, entre La Ribera y Añazco Pueblo, 1850 m, 1 Sep 2000 (fl, fr), 06 $84.705^{\prime}$ S 77²8.440'W, S.Leiva \& V.Quipuscoa 2470 (lectotype, designated in Deanna et al. 2014a, pg. 23: HUT! [40031, two sheets]).

Deprea longipedunculata (S.Leiva, E.Rodr. \& J.Campos) Barboza, comb. nov. urn:lsid:ipni.org:names:77145438-1

Basionym: Larnax longipedunculata S.Leiva, E.Rodr. \& J.Campos, Arnaldoa 5 (2): 194. 1998.

Type. PERU. Cajamarca: San Ignacio, Caserío La Bermeja, bosques de neblina La Bermeja, Dist. Tabaconas, 1830 m, 4 Jan 1998 (fl, fr), S.Leiva, J.Campos \& E.Rodríguez Rodriguez 2098 (lectotype, designated in Deanna et al. 2014a, pg. 25: CONN! [00055675]; isolectotypes: CORD! [00004044], F! [2198658], HUT! [031885], MO! [04906479]).

Deprea lutea (S.Leiva) Deanna, comb. nov. urn:lsid:ipni.org:names:77145439-1

Basionym: Larnax lutea S.Leiva, Arnaldoa 4 (1): 19. 1996.

Type. PERU. Cajamarca: Chota, $1 \mathrm{~km}$ del poblado de Paraguay (Querocoto-La Granja), 2250 m, 7 Aug 1994 (fl, fr), S.Leiva González, P.Chuna \& J.Cadle 1385 (lectotype, designated in Deanna et al. 2014a, pg. 25: CORD! [00004045]; isolectotype: F! [2177616]).

Deprea macasiana (Deanna, S.Leiva \& Barboza) Barboza, comb. nov. urn:lsid:ipni.org:names:77145421-1

Basionym: Larnax macasiana Deanna, S.Leiva \& Barboza, Phytotaxa 167 (1): 3.2014.

Type. ECUADOR. Morona Santiago: Macas, Cerro San José del Quílamo, $500 \mathrm{~m}$ antes de la Virgen Purísima de Macas en el Quílamo, 1369 m, 7808'19.3"W 02¹7'45.4"S, 23 Jan 2013 (fl, fr), R.Deanna \& S.Leiva 111 (holotype: QUSF! [29472]; isotypes: CORD! [00006797, 00006799], HAO!, QUSF! [29480]). 
Deprea maculatifolia (E.Rodr. \& S.Leiva) S.Leiva, comb. nov. urn:lsid:ipni.org:names:77145422-1

Basionym: Larnax maculatifolia E.Rodr. \& S.Leiva, Arnaldoa 9 (2): 102. 2003.

Type. PERU. Amazonas: Bagua, Imaza, Comunidad Aguaruna de Yamayakat, 390 m, 10 Jan 2001 (fl, fr), E.Rodríguez Rodríguez, S.Leiva \& R.Apanu 2384 (holotype: HUT! [38027]; isotypes: MO! [5763266], USM! [000899]).

Deprea nieva (S.Leiva \& N.W.Sawyer) Barboza \& Deanna, comb. nov. urn:lsid:ipni.org:names:77145440-1

Basionym: Larnax nieva S.Leiva \& N.W.Sawyer, Arnaldoa 10 (1): 106. 2003.

Type. PERU. Amazonas: Bongará, km 384 carretera Nueva Cajamarca-Pomacochas (Florida), 2000 m, 05.41 S, 77.46 W, 12 June 1997 (fl, fr), S.Leiva \& N.W.Sawyer 2045 (lectotype, designated in Deanna et al. 2014a, pg. 25: Leiva González \& Barboza, Arnaldoa 16(1): 32. Fig. 1. 2009).

Deprea parviflora (N.W.Sawyer \& S.Leiva) S.Leiva, comb. nov. urn:Isid:ipni.org:names:77145441-1

Basionym: Larnax parviflora N.W.Sawyer \& S.Leiva, Novon 11 (4): 466. 2001.

Type. PERU. Cajamarca: Cutervo, Bosque Cutervo, Parque Nacional Cutervo, NW border of Cordillera Tarros, Chorro Blanco sector, ca. $10 \mathrm{~km} \mathrm{WNW} \mathrm{of} \mathrm{San} \mathrm{Andres} \mathrm{de}$ Cutervo, ca. $2650 \mathrm{~m}, 6^{\circ} 12^{\prime} \mathrm{S}, 78^{\circ} 46^{\prime} \mathrm{W}, 4$ Nov 1990 (f), M.O.Dillon, I.Sanchez V. \& J.Guevara B. 6141 (holotype: F! [2059067]; isotype: CONN! [00168928]).

\section{Deprea pedrazae (S.Leiva \& Barboza) Deanna \& S.Leiva, comb. nov.} urn:lsid:ipni.org:names:77145423-1

Basionym: Larnax pedrazae S.Leiva \& Barboza, Arnaldoa 16 (2): 14. 2009.

Type. PERU. Amazonas: Bagua, La Peca, Puente El Arenal (ruta La Peca-El Arenal), 1170 m, 5³5'27.2"S, 78²4'20.8"W, 12 Oct 2009 (fl, fr), S.Leiva, M.Zapata \& G. Gayoso 4579 (lectotype, designated in Deanna et al. 2014a, pg. 25: Leiva González, Bravi \& Barboza, Arnaldoa 16(2): 16. Fig. 1. 2009). 
Deprea peruviana (Zahlbr.) S.Leiva \& Barboza, comb. nov. urn:lsid:ipni.org:names:77145424-1

Basionym: Athenaea peruviana Zahlbr., Ann. K. K. Naturhist. Hofmus. 7 (1-2): 7-8. 1892. Larnax peruviana (Zahlbr.) Hunz., Kurtziana 10: 9. 1977.

Type. [PERU. Cajamarca: Cutervo, La Ramada]. Tambillo, 29 July 1878 (fl, fr), C. de Jelski 54 (lectotype, designated in Deanna et al. 2014a, pg. 25: W! [1891-0004186]).

Deprea pilosa (S.Leiva, E.Rodr. \& J.Campos) Deanna, comb. nov. urn:lsid:ipni.org:names:77145442-1

Basionym: Larnax pilosa S.Leiva, E.Rodr. \& J.Campos, Arnaldoa 5 (2): 200. 1998.

Type. PERU. Cajamarca: San Ignacio, San José de Lourdes, Estrella del Oriente, borde de camino, 1600 m, 8 Jan 1998 (fl, fr), S. Leiva, J. Campos \& E. Rodriguez 2108 (lectotype, designated in Deanna et al. 2014a, pg. 26: CONN! [00051759]; isolectotypes: CORD! [00004047], F! [2198655], HUT! [031894], M! [M-0171580], MO! [04908631], NY! [00328792], USM! [000900]).

Deprea pomacochaensis (S.Leiva) Barboza, comb. nov. urn:lsid:ipni.org:names:77145425-1

Basionym: Larnax pomacochaensis S.Leiva, Arnaldoa 13 (2): 299. 2006.

Type. PERU: Amazonas: Bongará, km 328-329 carretera Bongará-Nueva Cajamarca, arriba de laguna Pomacochas, 2400 m, 5 Feb 2006 (fl, fr), S.Leiva 3542 (lectotype, designated in Deanna et al. 2014a, pg. 28: Leiva González \& Rodríguez Rodríguez, Arnaldoa 13(2): 300. Fig. 5. 2006).

Deprea psilophyta (N.W.Sawyer) S.Leiva \& Deanna, comb. nov. urn:lsid:ipni.org:names:77145443-1

Basionym: Larnax psilophyta N.W.Sawyer, Novon 8 (1): 74.1998.

Type. ECUADOR. Zamora-Chinchipe: Nudo de Sabanilla, pass on road from Yanganá to Valladolid, elfin forest and clearings, 2800-2900 m, 5 Apr 1985 (fl, fr), G.W.Harling \& L.Andersson 23724 (holotype: NY! [00312927]; isotype: QCA! [95-13/83]). 
Deprea pumila (S.Leiva, Barboza \& Deanna) S.Leiva, comb. nov. urn:Isid:ipni.org:names:77145426-1

Basionym: Larnax pumila S.Leiva, Barboza \& Deanna, Phytotaxa 167 (1): 8. 2014.

Type. ECUADOR. Pastaza: Mera, rumbo hacia Río Anzú, sendero, $1340 \mathrm{~m}$, 78 04'01.5"W, 0125'31.6"S, 13 Nov 2011 (fl, fr), C.I.Orozco, G.E.Barboza, A. Orejuela \& S.Leiva 3890 (holotype: CORD! [0006758]; isotypes: COL!, QCA!).

Deprea purpurea (S.Leiva) Barboza \& S.Leiva, comb. nov. urn:lsid:ipni.org:names:77145444-1

Basionym: Larnax purpurea S.Leiva, Arnaldoa 4 (1): 16. 1996.

Type. PERU. Cajamarca: San Ignacio, Ruta San Ignacio-El Chaupe, riachuelo, entre abundantes arbustos, $1510 \mathrm{~m}$, 4 Jan 1995 (fl, fr), S.Leiva G., P.Lezama A. \& P.Chuna 1560 (lectotype, designated in Deanna et al. 2014a, pg. 29: CORD! [00004049]; isolectotypes: F! [2177617], HUT! [031931], MO! [05077371], NY! [00076787], USM! [000901]).

Deprea purpureocarpa (S.Leiva, Deanna \& Barboza) Deanna, comb. nov. urn:Isid:ipni.org:names:77145427-1

Basionym: Larnax purpureocarpa S.Leiva, Deanna \& Barboza, Phytotaxa 167 (1): 12. 2014.

Type. ECUADOR. Napo: carretera Cosanga-Baeza, $5.4 \mathrm{~km}$ al sur de Baeza, en borde de carretera, $1855 \mathrm{~m}, 77^{\circ} 52^{\prime} 20.2^{\prime \prime W}, 00^{\circ} 28^{\prime} 34.2^{\prime \prime S}, 25$ Jan 2013 (fl, fr), R.Deanna \& S.Leiva 125 (holotype: QCNE! [0233491]; isotypes: CORD! [00006800], HAO!).

Deprea sachapapa (Hunz.) S.Leiva \& Deanna, comb. nov. urn:lsid:ipni.org:names:77145445-1

Basionym: Larnax sachapapa Hunz., Kurtziana 10: 13-15. 1977.

Type. ECUADOR. Azuay: between Cruz Pamba and Loma de Canela, in region of Río Sadracay, tributary of Río Mehuir, north of Molleturo, in Cinchona forest, 23152500 m, 12 June 1943 (fl, fr), J.A.Steyermark 52965 (holotype: VEN! [119697]; isotypes: CORD! [00006739], F! [1276519]). 
Deprea sagasteguii (S.Leiva, Quip. \& N.W.Sawyer) Barboza, comb. nov. urn:lsid:ipni.org:names:77145428-1

Basionym: Larnax sagasteguii S.Leiva, Quip. \& N.W.Sawyer, Arnaldoa 5 (1): 86-89. 1998.

Type. PERU. Piura: Ayabaca, Cerro Aypate, 2800-2880 m, 442.94'S, 79³4.25'W, 23 May 1996 (fl, fr), V.Quipuscoa S., O.Angulo Z. \& R.Yahuana 601 (lectotype designated in Deanna et al. 2014a, pg. 30: HUT! [031934]).

Deprea sawyeriana (S.Leiva, E.Rodr. \& J.Campos) S. Leiva, comb. nov. urn:lsid:ipni.org:names:77145446-1

Basionym: Larnax sawyeriana S.Leiva, E.Rodr. \& J.Campos, Arnaldoa 5 (2): 203. 1998.

Type. PERU. Cajamarca: San Ignacio, Caserío La Bermeja, bosques de neblina La Bermeja, Dist. Tabaconas, 1830 m, 4 Jan 1998 (fl, fr), S.Leiva, J.Campos \& E.Rodríguez 2097 (lectotype, designated in Deanna et al. 2014a, pg. 31: HUT! [031884]; isolectotypes: CONN! [00055668], CORD! [00004051], F! [2198659], MO! [04906480]).

Deprea schjellerupiae (S.Leiva \& Quip.) Barboza \& Deanna, comb. nov. urn:lsid:ipni.org:names:77145447-1

Basionym: Larnax schjellerupiae S.Leiva \& Quip., Arnaldoa 9 (1): 32.2002 [2003].

Type. PERU. San Martin: Huallaga, La Fila, entre Añazco Pueblo y Leguía, 2000 m, 10 Sep 2000 (fr), S.Leiva \& V.Quipuscoa 2479 (lectotype, designated in Deanna et al. 2014a, pg. 31: HUT! [40030]).

Deprea steyermarkii (Hunz.) S.Leiva \& Barboza, comb. nov. urn:lsid:ipni.org:names:77145448-1

Basionym: Larnax steyermarkii Hunz., Kurtziana 10: 11. 1977.

Type. ECUADOR. Zamora: Arenillas, at junction of río Santa Bárbara and río Tintas, between Ca mpanas and Arenillas, southeast of El Pan, 2195 m, 13 July 1943 (fl, fr), J.A.Steyermark 53535 (holotype: VEN! [119698]; isotypes: CORD!, F! [1276523, 1739588]). 
Deprea toledoana (Barboza \& S.Leiva) Barboza, comb. nov. urn:Isid:ipni.org:names:77145429-1

Basionym: Larnax toledoana Barboza \& S.Leiva, Phytotaxa 167 (1): 16.2014.

Type. ECUADOR. Zamora-Chinchipe: [Cerro Toledo], rumbo a Valladolid desde Yanganá, al costado de la ruta, $2690 \mathrm{~m}, 79^{\circ} 08^{\prime} 52.7^{\prime \prime W}, 04^{\circ} 26^{\prime} 30.7^{\circ} \mathrm{S}, 16$ Nov 2011 (fl, fr), C.I.Orozco, G.E.Barboza, A.Orejuela \& S.Leiva 3936 (holotype: QCA!; isotypes: CORD! [00006769, 00006770, 00006771], COL!, HAO!).

\section{Deprea vasquezii (S.Leiva, E.Rodr. \& J.Campos) Deanna, comb. nov.} urn:Isid:ipni.org:names:77145449-1

Basionym: Larnax vasquezii S.Leiva, E.Rodr. \& J.Campos, Arnaldoa 5 (2): 206.1998.

Type. PERU. Cajamarca: San Ignacio, Dist. San José de Lourdes, Estrella del Oriente, $1600 \mathrm{~m}, 04^{\circ} 50^{\prime} \mathrm{S}, 78^{\circ} 55^{\prime} \mathrm{W}, 8$ Jan 1998 (fl, fr), S.Leiva, J.Campos \& E.Rodríguez 2109 (lectotype, designated in Deanna et al. 2014a, pg. 31: US! [00623565]; isolectotypes: CONN! [00055674], CORD! [00004050], F! [2198657], M! [M-0171574], MO! [04908632], NY! [00328793], US! [00623565], USM! [000902]).

After these new combinations, the genus Deprea includes the following 45 currently accepted species:

1. Deprea abra-patriciae (S.Leiva \& Barboza) S.Leiva \& Deanna

2. Deprea altomayoensis (S.Leiva \& Quip.) Barboza \& Deanna

3. Deprea andersonii (N.W.Sawyer) Deanna \& S.Leiva

4. Deprea bitteriana (Werderm.) N.W.Sawyer \& Benítez

5. Deprea bongaraensis (S.Leiva) Deanna \& Barboza

6. Deprea cardenasiana Hunz.

7. Deprea chotanae (S.Leiva, Pereyra \& Barboza) S.Leiva

8. Deprea cuyacensis (N.W.Sawyer \& S.Leiva) S.Leiva \& Lezama

9. Deprea cyanocarpa J.Garzon \& C.I.Orozco

10. Deprea darcyana (N.W.Sawyer) Barboza \& S.Leiva

11. Deprea dilloniana (S.Leiva, Quip. \& N.W.Sawyer) Barboza

12. Deprea ecuatoriana Hunz. \& Barboza

13. Deprea glabra (Standl.) Hunz.

14. Deprea grandiflora (N.W.Sawyer \& S.Leiva) Deanna \& Barboza

15. Deprea harlingiana (Hunz. \& Barboza) S.Leiva \& Deanna

16. Deprea hawkesii (Hunz.) Deanna

17. Deprea kann-rasmussenii (S.Leiva \& Quip.) S.Leiva \& Barboza 
18. Deprea longipedunculata (S.Leiva, E.Rodr. \& J.Campos) Barboza

19. Deprea lutea (S.Leiva) Deanna

20. Deprea macasiana (Deanna, S.Leiva \& Barboza) Barboza

21. Deprea maculatifolia (E.Rodr. \& S.Leiva) S.Leiva

22. Deprea nieva (S.Leiva \& N.W.Sawyer) Barboza \& Deanna

23. Deprea nubicola N.W.Sawyer

24. Deprea orinocensis (Kunth) Raf.

25. Deprea oxapampensis M.Cueva \& I.Treviño

26. Deprea paneroi Benítez \& M.Martínez

27. Deprea parviflora (N.W.Sawyer \& S.Leiva) S.Leiva

28. Deprea pedrazae (S.Leiva \& Barboza) Deanna \& S.Leiva

29. Deprea peruviana (Zahlbr.) S.Leiva \& Barboza

30. Deprea pilosa (S.Leiva, E.Rodr. \& J.Campos) Deanna

31. Deprea pomacochaensis (S.Leiva) Barboza

32. Deprea psilophyta (N.W.Sawyer) S.Leiva \& Deanna

33. Deprea pumila (S.Leiva, Barboza \& Deanna) S.Leiva

34. Deprea purpurea (S.Leiva) Barboza \& S.Leiva

35. Deprea purpureocarpa (S.Leiva, Deanna \& Barboza) Deanna

36. Deprea sachapapa (Hunz.) S.Leiva \& Deanna

37. Deprea sagasteguii (S.Leiva, Quip. \& N.W.Sawyer) Barboza

38. Deprea sawyeriana (S.Leiva, E.Rodr. \& J.Campos) S. Leiva

39. Deprea schjellerupiae (S.Leiva \& Quip.) Barboza \& Deanna

40. Deprea steyermarkii (Hunz.) S.Leiva \& Barboza

41. Deprea subtriflora (Ruiz \& Pav.) D’Arcy

42a. Deprea sylvarum (Standl. \& C.V.Morton) Hunz. subsp. sylvarum

42b. Deprea sylvarum (Standl. \& C.V.Morton) Hunz. subsp. novogranatensis N.W.Sawyer

43. Deprea toledoana (Barboza \& S.Leiva) Barboza

44. Deprea vasquezii (S.Leiva, E.Rodr. \& J.Campos) Deanna

45. Deprea zamorae Barboza \& S.Leiva

\section{Acknowledgements}

The authors thank the IMBIV-CONICET (Cordoba, Argentina), the Universidad Nacional de Córdoba (Argentina), and the Universidad Privada Antenor Orrego (Trujillo, Peru) for their facilities to perform this study; the Consejo Nacional de Investigaciones Científicas y Técnicas (CONICET, PID 00552), Fondo para la Investigación Científica y Tecnológica (FONCYT, PICT 2775), and the Secretaría de Ciencia y Tecnología, Universidad Nacional de Córdoba (SECyT-UNC) from Argentina for funding the study. We are also indebted to the reviewers for valuable suggestions and comments on the manuscript. The first author would like to especially thank FONCYT and CONICET for the scholarships provided for the doctorate studies. 


\section{References}

Barboza GE, Hunziker AT (1994) Estudios sobre Solanaceae XXXVII. Sinopsis taxonómica de Deprea. Kurtziana 23: 101-124.

Cardona D, Quiñones W, Torres F, Vélez ID, Orozco CI, Garzón J, Echeverri F (2005) Estructura y actividad leishmanicida de larnaxolida A y B, nuevos withanólidos de Larnax glabra (Standl.) Sawyer. Actualidades Biológicas 27: 81-86.

Carrizo García C, Wahlert G, Orozco CI, Barboza GE, Bohs L (submitted) Phylogeny of the Andean genus Deprea (Physalideae, Solanaceae): testing the generic circumscription. Phytotaxa. doi: 10.1016/j.phytochem.2014.11.015

Casero CN, Oberti JC, Orozco CI, Cárdena A, Brito I, Barboza GE, Nicotra VE (2015) Withanolides from three species of the genus Deprea (Solanaceae). Chemotaxonomical considerations. Phytochemistry 110: 83-90. doi: 10.1016/j.phytochem.2014.11.015

D’Arcy WG (1973) New names for Neotropical plants. Phytologia 25: 116.

D’Arcy WG (1993) A new combination in Deprea (Solanaceae). Novon 3: 22. doi: $10.2307 / 3391412$

Deanna R, Leiva González S, Barboza GE(2014a) Four new species and eighteen lectotypifications of Larnax from Ecuador and Peru and a new synonym of Deprea orinocensis (Solanaceae: Solanoideae, Physalideae). Phytotaxa 167(1): 1-34. doi: 10.11646/phytotaxa.167.1.1

Deanna R, Barboza GE, Scaldaferro MA (2014b) First karyological report in Larnax and Deprea. Australian Journal of Botany 62: 251-261. doi: 10.1071/BT14041

Jørgensen PM, León-Yánez S (1999) Catálogo de las plantas vasculares del Ecuador. Monographs in Systematic Botany from the Missouri Botanical Garden 75: 1-1181.

Hunziker AT (2001) Genera Solanacearum. A. R. G. Gantner Verlag K.-G., Ruggell, 1-500.

Leiva González S, Deanna R, Barboza GE, Cueva Manchego M (2013) Sobre la presencia del género Larnax (Solanaceae) en Bolivia. Arnaldoa 20(2): 291-300.

Leiva González S, Lezama Asencio P, Zapata Cruz M (2005) Primera especie de Deprea Rafinesque (Solanaceae: Solaneae) en Perú. Arnaldoa 12 (1-2): 62-66.

Leiva González S, Pereyra Villanueva E, Barboza GE (2008) Larnax altomayoensis y Larnax chotanae (Solanaceae) dos nuevas especies de los bosques montanos del Norte del Perú. Arnaldoa 15: 197-209.

Miers J (1849) Contributions to the botany of South America. Annals and Magazine of Natural History, ser II, 4: 31-39. doi: 10.1080/03745486009494796

Misico RI, Nicotra V, Oberti JC, Barboza G, Gil RR, Burton G (2011) Withanolides and related steroids. Progress in the Chemistry of Organic Natural Products 94: 127-229. doi: 10.1007/978-3-7091-0748-5_3

Olmstead R, Bohs L, Migid HA, Santiago-Valentín E, García VF, Collier SM (2008) A molecular phylogeny of the Solanaceae. Taxon 57: 1159-1181. http://depts.washington.edu/ phylo/OlmsteadPubs/Olmstead_et_al_2008.pdf

Särkinen T, Bohs L, Olmstead RG, Knapp S (2013) A phylogenetic framework study of the nightshades (Solanaceae): a dated 1000-tip tree. BMC Evolutionary Biology 13: 214-229. doi: $10.1186 / 1471-2148-13-214$ 
Sawyer NW (1999) The systematics of Deprea Raf. And Larnax (Miers) Hunz. (Solanaceae). PhD Thesis, University of Connecticut, Storrs, CT.

Sawyer NW (2001) New species and combination in Larnax (Solanaceae). Novon 11: 460-471. doi: $10.2307 / 3393161$

Sawyer NW (2005) Systematics of Deprea and Larnax (Solanaceae) based on morphological evidence. In: Keating RC, Hollowell VC, Croat TB (Eds) A festschrift for William G. D’Arcy. Missouri Botanical Garden, Saint Louis, 259-285. 